\title{
The effects of pentoxifylline and tocopherol in jaw osteomyelitis
}

\author{
Mi Hyun Seo, Mi Young Eo, Hoon Myoung, Soung Min Kim, Jong Ho Lee \\ Department of Oral and Maxillofacial Surgery, School of Dentistry and Dental Research Institute, \\ Seoul National University, Seoul, Korea
}

\begin{abstract}
J Korean Assoc Oral Maxillofac Surg 2020;46:19-27)
Objectives: Pentoxifylline (PTX) is a methylxanthine derivative that has been implicated in the pathogenesis of peripheral vessel disease and intermittent lameness. The purpose of this study was to investigate the effect of PTX and tocopherol in patients diagnosed with osteoradionecrosis (ORN), bisphosphonate-related osteonecrosis of the jaw (BRONJ), and chronic osteomyelitis using digital panoramic radiographs.

Materials and Methods: This study was performed in 25 patients who were prescribed PTX and tocopherol for treatment of ORN, BRONJ, and chronic osteomyelitis between January 2014 and May 2018 in Seoul National University Dental Hospital. Radiographic densities of the dental panorama were compared prior to starting PTX and tocopherol, at 3 months, and at 6 months after prescription. Radiographic densities were measured using Adobe Photoshop CS6 (Adobe System Inc., USA). Blood sample tests showing the degree of inflammation at the initial visit were considered the baseline and compared with results after 3 to 6 months. Statistical analysis was performed using the Mann-Whitney test and repeated measurement ANOVA using IBM SPSS 23.0 (IBM Corp., USA).

Results: Eight patients were diagnosed with ORN, nine patients with BRONJ, and the other 8 patients with chronic osteomyelitis. Ten of the 25 patients were men, average age was $66.32 \pm 14.39$ years, and average duration of medication was $151.8 \pm 80.65$ days (range, $56-315$ days). Statistically significant increases were observed in the changes between 3 and 6 months after prescription $(P<0.05)$. There was no significant difference between ORN, BRONJ, and chronic osteomyelitis. Only erythrocyte sedimentation rate (ESR) was statistically significantly lower than before treatment $(P<0.05)$ among the white blood cell (WBC), ESR, and absolute neutrophil count (ANC).

Conclusion: Long-term use of PTX and tocopherol can be an auxiliary method in the treatment of ORN, BRONJ, or chronic osteomyelitis in jaw.
\end{abstract}

Key words: Pentoxifylline, Tocopherols, Panoramic radiography, Osteomyelitis

[paper submitted 2018. 9. 29 / revised 2018. 10. 9 / accepted 2018. 10. 9]

\section{Introduction}

Pentoxifylline [1-(5-oxohexyl)-3,7-dimethylxanthine, PTX] is a methylxanthine derivative ${ }^{1}$ that has been implicated in the pathogenesis of peripheral vessel disease and intermittent lameness ${ }^{2}$. PTX is a non-selective inhibitor of cyclic nucleotide phosphodiesterase (PDE) that inhibits cyclic adenosine monophosphate (cAMP) PDE, increases cAMP and

\footnotetext{
Soung Min Kim Jongno-gu, Seoul 03080, Korea

TEL: +82-2-2072-0213 FAX: +82-2-766-4948

E-mail:smin5@snu.ac.kr

ORCID: https://orcid.org/0000-0002-6916-0489
}

Department of Oral and Maxillofacial Surgery, School of Dentistry and Dental Research Institute, Seoul National University, 101 Daehak-ro,

(c) This is an open-access article distributed under the terms of the Creative Commons Attribution Non-Commercial License (http://creativecommons.org/ licenses/by-nc/4.0/), which permits unrestricted non-commercial use, distribution, and reproduction in any medium, provided the original work is properly cited. Copyright (C) 2020 The Korean Association of Oral and Maxillofacial Surgeons. All rights reserved. adenosine-5'-triphosphate in erythrocytes, and increases red blood cell deformability. PTX decreases leukocyte adhesion to endothelial cells, increases prostacyclin production, and inhibits platelet aggregation. These effects induce capillary dilatation, reduce blood viscosity, and improve peripheral blood flow ${ }^{3}$. Recently, PTX has been shown to exhibit immunomodulatory functions to downregulate the production of proinflammatory cytokines, particularly tumor necrosis factor alpha $(\mathrm{TNF}-\alpha)^{4}$.

Tocopherols are a class of organic chemical compounds consisting of various methylated phenols. These compounds possess antioxidant effects to protect cell membranes from lipid peroxidation and are known to partially inhibit transforming growth factor- $\beta 1$. Tocopherols also reduce inflammation and tissue fibrosis. The combination of PTX and tocopherol has synergistic effects, but the mechanism of action remains unclear ${ }^{5}$.

The effects of PTX and tocopherol have been reported to be encouraging in the treatment of osteoradionecrosis 
$(\mathrm{ORN})^{6,7}$. There are some reports that the combination of PTX and tocopherol is effective against bisphosphonaterelated osteonecrosis of the jaw (BRONJ).

ORN, BRONJ, and chronic osteomyelitis have similar clinical characteristics, yet are different in pathophysiology. The three disease entities are clinically similar and may represent oral bone exposure that cannot be cured within an 8-week period $^{8}$. Various classification systems have been proposed for these disease entities. In the staging of osteomyelitis, Notani's classification of ORN was used in this study. In a panorama image, stage I is defined as lesions restricted to the alveolar bone, stage II is defined as lesions restricted to the alveolar bone and/or above the inferior alveolar canal, while stage III was defined as the invasion to the lower part of the inferior alveolar canal, extraoral fistulae, or pathologic bone fracture? BRONJ patients are defined as those with a history of treatment with bisphosphonates, bone exposure for eight weeks or longer, and no history of radiation therapy (RT) or evidence of metastatic disease to the jaws ${ }^{10}$. Patients without a history of RT or antiresorptive drugs such as bisphosphonates were classified as chronic osteomyelitis. The BRONJ group was classified according to the BRONJ staging system with 2014 American Association of Oral and Maxillofacial Surgeons (AAOMS) guidelines ${ }^{10,11}$.

Basic images involve periapicals and panoramas. Computed tomography creates three dimensional images and accurately shows the extension of lesions. Panoramic radiographs are routinely performed during diagnosis and follow-up and allow for easy comparisons of bony changes following treatment. In this study, the effects of PTX and tocopherol were evaluated by serial panoramic analysis of patients diagnosed with ORN, BRONJ, or chronic osteomyelitis in jaw.

\section{Materials and Methods}

We retrospectively analyzed 25 patients who were prescribed PTX and tocopherol for treatment of ORN, BRONJ, or chronic osteomyelitis of the mandible between January 2014 and May 2018 in Seoul National University Dental Hospital. All patients received a combination of $800 \mathrm{mg}$ of PTX and 800 IU of vitamin E every day. The PTX dosage was designed to avoid severe adverse effects in patients without vascular disease, while the vitamin E dosage was determined to supply sufficient antioxidant activity. There were no serious side effects during the study period. All patients were divided into two groups: PTX and tocopherol for less than 90 days (short-term), and group for more than 90 days (longterm). While the effect of PTX may be seen within 2 to 4 weeks, it is recommended that treatment be continued at least 8 weeks $^{12}$.

We evaluated serial dental panoramas using Adobe Photoshop CS6 (Adobe System Inc., San Jose, CA, USA). The Orthopantomograph OP100 (Instrumentaruim Corp., Helsinki, Finland) was used for panoramic radiographs. By setting the region of interest (ROI) as the radiolucent defect area in dental panoramic views and drawing the contour of the bone defect using the selection tool of Adobe Photoshop CS6, the average densities of the selected area could be obtained as pixel values.(Fig. 1) We analyzed the panoramic views taken before prescription and three and six months after the first prescription. One observer measured the densities of the ROI and contralateral side and used the average value of the three measurements. Clinical photographs were taken at the same time point as the radiographs, and the degree of densities in the radiographs was compared with the clinical characteristics. Bony healing was evaluated by two methods: the first method involved measurement of radiographic densities of ROI, while the second method calculated the ratio of the densities of ROI to the opposite side. Densities of the contralateral side to the ROI was measured by the same method and set as the reference value. Using the selection tool of Photoshop CS6, the same dimension was designated for analysis with reference to the number of pixels of the selected area. The teeth and foreign body were excluded from the ROI and

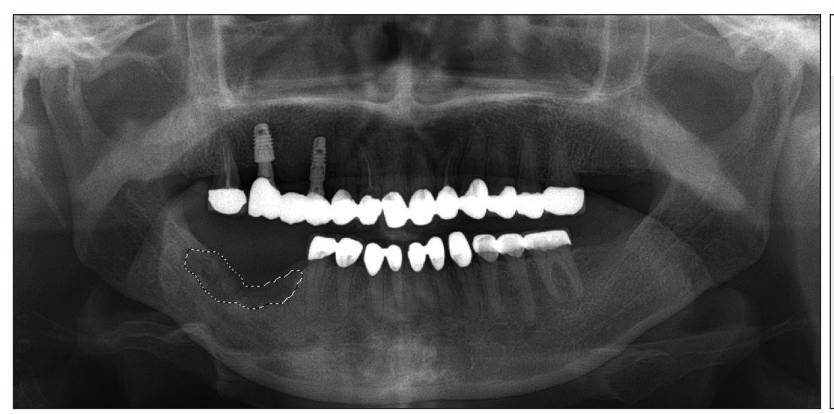

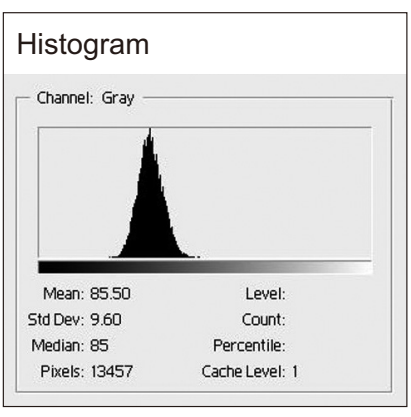

Fig. 1. The method used in this study to quantify the densities of defects in the panoramic views. These analyses were performed using Adobe Photoshop CS6 Histogram (Adobe System Inc., USA).

Mi Hyun Seo et al: The effects of pentoxifylline and tocopherol in jaw osteomyelitis. J Korean Assoc Oral Maxillofac Surg 2020 
only the bone area was evaluated. The differences of densities between ROI and the reference value were used for statistical analysis with the IBM SPSS 23.0 program (IBM Corp., Armonk, NY, USA). The Mann-Whitney test and repeated measures ANOVA were used in the study, and the results were considered statistically significant at $P<0.05$.

Initial complete blood count (CBC) and erythrocyte sedimentation rate (ESR) were statistically analyzed between disease groups using the Kruskal-Wallis test. The Bonferroni correction was used to modify the alpha level, and statistical significance was considered at $P<0.05$.

We compared the blood sample tests for baseline and follow up data after three to six months. The inflammatory markers of white blood cell (WBC), ESR, and absolute neutrophil count (ANC) were compared according to time, duration of PTX and tocopherol usage, and disease entity. Statistical analysis was performed with repeated measures ANOVA, and the results were considered statistically significant at $P<0.05$.

The collection and processing of this retrospective clinical data were approved by the Institutional Review Board of Seoul National University (IRB No. S-D20160041).

\section{Results}

Among the 25 patients, 8 were diagnosed with ORN, 9 were diagnosed with BRONJ, and 8 were diagnosed with chronic osteomyelitis.(Table 1) Ten of the 25 patients were men; however, the proportion of women was significantly higher in the BRONJ group, 8 of 9 patients. The average age was $66.32 \pm 14.39$ with a duration of medication of 151.8 \pm 80.65 days (range, 56-315 days). Two patients received only medical treatment with one patient receiving RT to cure tonsillar cancer 8.3 years prior to refer from a local clinic for an unhealed extraction socket diagnosed as ORN. The patient was treated with PTX and tocopherol for approximately 10 months, and radiographic densities increased in the panoramic view. Clinical photos were taken at the same time as the radiographs. One year after PTX and tocopherol usage, soft tissue healing was observed without bony exposure.(Fig. 2) The other patient was diagnosed with squamous cell carcinoma on the lower gingiva and treated with partial mandibulectomy, partial maxillectomy, and selective neck dissection and reconstruction with a radial forearm free flap. Postoperative RT was performed for near resection margins and perineural invasion. Radiographic change was observed during osteotomy of the mandible with the swing approach. The patient was treated with PTX and tocopherol for approximately 6 months. Other patients, except two, underwent surgical treatment such as curettage, sequestrectomy, saucerization, and initiation of PTX and tocopherol when the surgical site became stable before beginning administration. Fig. 3 shows serial radiographic changes of the patient (Case No. 7) with ORN. This patient received surgical intervention including extraction and saucerization. After wound healing, PTX and tocopherol were prescribed for 56 days. Six months after drug administration, the radiographic bony densities decreased and osteolytic bony lesions were extended. However, in the case diagnosed with Stage 3 BRONJ, long-term use of PTX and tocopherol with surgery produced good bony healing.(Fig. 4) Osteolytic lesions that extended to the mandibular border were diminished.

We compared the radiographic changes in the panoramic view at T0 (before the start of medication), T1 (3 months after medication), and T2 (6 months after medication). We divided the experimental group according to duration of medication usage and type of disease. Statistically significant increases were observed in the changes in T2-T1 $(\Delta 2)$ and T2-T0 $(\Delta 3)$ in those taking the drug in excess of 90 days $(P<0.05)$. (Table 2) There was no significant difference between diseases of ORN, BRONJ, and chronic osteomyelitis. Changes of radiographic densities compared to the reference site were also evaluated. Difference of densities between the ROIs and the contralateral sites was divided by the density of the contralateral sites. The percentages were evaluated before drug administration. Three and six months after administration, the differences of radiographic densities compared to the contralateral sites exhibited statistically significant reductions $(P<0.05)$. However, there was no statistical difference between the disease groups.(Table 3 )

We collected laboratory data including WBC, hemoglobin $(\mathrm{Hb})$, hematocrit (Hct), ANC, and ESR at the initial hospital visit. Statistically significant differences were found in $\mathrm{Hb}$ between the ORN and BRONJ groups $(P=0.016)$. In the laboratory test of inflammatory markers, only ESR exhibited statistically significant changes with time $(P<0.05)$.(Table 4$)$ There were no statistically significant differences according to disease group or duration of drug usage over time.

\section{Discussion}

The efficacy of PTX appeared within 2 to 4 weeks, and it is recommended that this drug be used for at least 8 weeks. Efficacy has been demonstrated in a double-blind trial for 6 
Table 1. Summary of the cases of ORN, BRONJ, or chronic osteomyelitis treated with PTX and tocopherol

\begin{tabular}{|c|c|c|c|c|c|c|c|c|c|c|c|}
\hline $\begin{array}{l}\text { Case } \\
\text { No. }\end{array}$ & Sex & $\begin{array}{l}\text { Age } \\
(\mathrm{yr})\end{array}$ & Disease & $\begin{array}{c}\text { Duration } \\
\text { of drug } \\
\text { usage } \\
\text { (day) }\end{array}$ & $\begin{array}{l}\text { Related past } \\
\text { medical history }\end{array}$ & Staging & $\begin{array}{l}\text { Radiation dose } \\
\text { (Gy)/causative } \\
\text { medication }\end{array}$ & $\begin{array}{l}\text { Initiation } \\
\text { factor }\end{array}$ & $\begin{array}{c}\text { Onset } \\
(\mathrm{yr})\end{array}$ & Site & Intervention \\
\hline 1 & M & 84 & ORN & 90 & $\mathrm{ACC}$ on $\mathrm{SMG}$ & 2 & 66 & Ext. & 4.61 & \#35 & Sequestrectomy \\
\hline 2 & $\mathrm{~F}$ & 42 & ORN & 315 & Tonsillar cancer & 2 & - & Ext. & 8.3 & \#46,47 & Medication only \\
\hline 3 & M & 60 & ORN & 140 & $\begin{array}{l}\text { SCC on lower } \\
\text { gingiva }\end{array}$ & 3 & 60 & Ext. & 0.62 & Angle & OR \& IF \\
\hline 4 & M & 55 & ORN & 174 & $\begin{array}{l}\text { SCC on lower } \\
\text { gingiva }\end{array}$ & 3 & 63 & Osteotomy & 0.4 & Body & Medication only \\
\hline 5 & $\mathrm{~F}$ & 67 & ORN & 56 & $\begin{array}{l}\text { ACC on parotid } \\
\text { gland }\end{array}$ & 1 & 70 & Ext. & 5.74 & $\# 34,35,36,37$ & Saucerization \\
\hline 6 & $\mathrm{~F}$ & 71 & ORN & 98 & $\begin{array}{l}\text { ACC on lower } \\
\text { lip }\end{array}$ & 2 & 72 & Ext. & 4.07 & $\begin{array}{l}\text { Anterior } \\
\text { mandible }\end{array}$ & Saucerization \\
\hline 7 & M & 86 & ORN & 56 & SCC on tongue & 2 & 70 & - & - & $\begin{array}{l}\text { Anterior } \\
\text { mandible }\end{array}$ & - \\
\hline 8 & $\mathrm{~F}$ & 57 & ORN & 252 & $\begin{array}{l}\text { SCC on } \\
\text { retromolar } \\
\text { trigone }\end{array}$ & 1 & 63 & - & 1.38 & \#37 & Saucerization \\
\hline 9 & $\mathrm{~F}$ & 61 & BRONJ & 320 & Osteoporosis & 2 & Alendronate & - & - & \#45,46,47 & Saucerization \\
\hline 10 & $\mathrm{~F}$ & 79 & BRONJ & 237 & Osteoporosis & 3 & - & Ext. & - & $\# 46,47$ & Sequestrectomy \\
\hline 11 & $\mathrm{~F}$ & 83 & BRONJ & 230 & Osteoporosis & 2 & $\begin{array}{l}\text { Risedronate, } \\
\text { alendronate }\end{array}$ & Ext. & - & $\# 44,45,46$ & Saucerization \\
\hline 12 & $\mathrm{~F}$ & 71 & BRONJ & 112 & Osteoporosis & 2 & - & Ext. & - & $\# 36,37$ & Saucerization \\
\hline 13 & $\mathrm{~F}$ & 86 & BRONJ & 84 & Osteoporosis & 2 & Ibandronate & Ext. & - & $\# 45,46,47$ & Saucerization \\
\hline 14 & $\mathrm{~F}$ & 70 & BRONJ & 56 & $\begin{array}{l}\text { Multiple } \\
\text { myeloma }\end{array}$ & 2 & - & - & - & $\begin{array}{l}\# 37,38 \\
\text { ascending } \\
\text { ramus }\end{array}$ & Saucerization \\
\hline 15 & $\mathrm{M}$ & 84 & BRONJ & 168 & Osteoporosis & 2 & Ibandronate & Ext. & - & $\# 46,47$ & Saucerization \\
\hline 16 & $\mathrm{~F}$ & 69 & BRONJ & 178 & Osteoporosis & 2 & Alendronate & - & - & $\# 47,48$ & Saucerization \\
\hline 17 & $\mathrm{~F}$ & 82 & BRONJ & 119 & Osteoporosis & 2 & Alendronate & Ext. & - & $\# 35$ & Saucerization \\
\hline 18 & $\mathrm{M}$ & 79 & $\mathrm{OM}$ & 180 & - & 1 & - & - & - & $\# 37,38$ & Sequestrectomy \\
\hline 19 & $\mathrm{~F}$ & 50 & $\mathrm{OM}$ & 252 & - & 2 & - & - & - & $\# 44,45,46$ & Saucerization \\
\hline 20 & $\mathrm{~F}$ & 50 & $\mathrm{OM}$ & 174 & - & 2 & - & - & - & $\# 35,36,37$ & $\begin{array}{l}\text { Ext., } \\
\text { sequestrectomy }\end{array}$ \\
\hline 21 & M & 56 & $\mathrm{OM}$ & 168 & - & 2 & - & - & - & $\# 47,48$ & Ext., saucerization \\
\hline 22 & $\mathrm{M}$ & 64 & $\mathrm{OM}$ & 140 & - & 3 & - & - & - & \#38 & Saucerization \\
\hline 23 & $\mathrm{M}$ & 41 & OM & 84 & - & 2 & - & - & - & \#47 & Sequestrectomy \\
\hline 24 & $\mathrm{~F}$ & 67 & $\mathrm{OM}$ & 56 & - & 1 & - & - & - & \#47 & Saucerization \\
\hline 25 & M & 44 & $\mathrm{OM}$ & 56 & - & 3 & - & - & - & \#48 & Saucerization \\
\hline
\end{tabular}

(ORN: osteoradionecrosis, BRONJ: bisphosphonate-related osteonecrosis of the jaw, PTX: pentoxifylline, Onset: the time from the end of radiotherapy to the diagnosis of ORN, M: male, F: female, ACC: adenoid cystic carcinoma, SMG: submandibular gland, Ext.: extraction, SCC: squamous cell carcinoma, OR \& IF: open reduction and internal fixation, OM: chronic osteomyelitis)

Mi Hyun Seo et al: The effects of pentoxifylline and tocopherol in jaw osteomyelitis. J Korean Assoc Oral Maxillofac Surg 2020

months ${ }^{12}$. Radiography is the major non-invasive method for detecting bone healing. In general, bone healing results in an increase in radiopacity, leading to high optical density ${ }^{13}$. Therefore, we used panoramic radiographs for evaluation of the effect of PTX and tocopherol in jaw osteomyelitis. In this study, there was a significant increase in radiographic densities in the group administered the drugs for more than 90 days compared to the group that had less than 90-days of administration for a period of 6 months after administration of PTX and tocopherol. At three months, both groups exhibited an increase in radiographic densities. However, between three and six months after administration of drugs (T2-T1, $\Delta 2$ ), the radiographic densities significantly increased in the group that had more than 90 days of administration of the drugs. Since surgical procedures such as curettage, sequestrectomy, and saucerization involve removal of partially pathological bone tissue, the degree of radiographic densities was reduced by surgery. Except for two cases, administration of drugs was initiated after wound stabilization. Therefore, over three months, bone healing occurred after the surgical procedure, and radiographic densities increased in most of the cases. After the initial healing period of surgery, continuous administration of the combination of PTX and tocopherol had a positive effect on bone healing. Not only did the degree of densities of the panorama increase, but intraoral bone exposures and inflammation clinically decreased.(Fig. 2. D, 4. B) There was no significant difference in degree of increase in radiographic densities based on disease. 

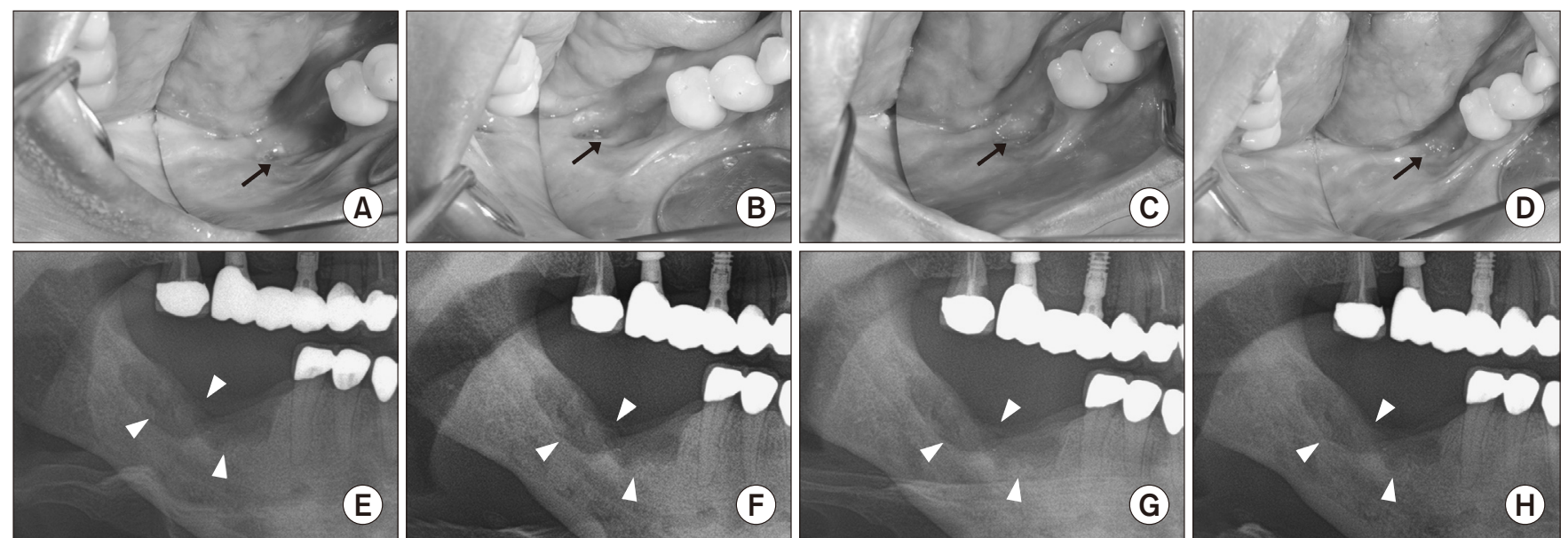

Fig. 2. Serial occlusal clinical photos and panoramic views after using pentoxifylline and tocopherol in the Case No. 2 patient who had not undergone any surgery and had taken medication for osteoradionecrosis for 315 days. A, E. Before prescription. B, F. Three months after drug administration. C, G. Six months after drug administration. D, H. One year after drug administration. (Arrows: lesion with intra-oral bone exposure, decreased over time; Arrowheads: osteoradionecrosis observed in the panoramic radiographs)

Mi Hyun Seo et al: The effects of pentoxifylline and tocopherol in jaw osteomyelitis. J Korean Assoc Oral Maxillofac Surg 2020
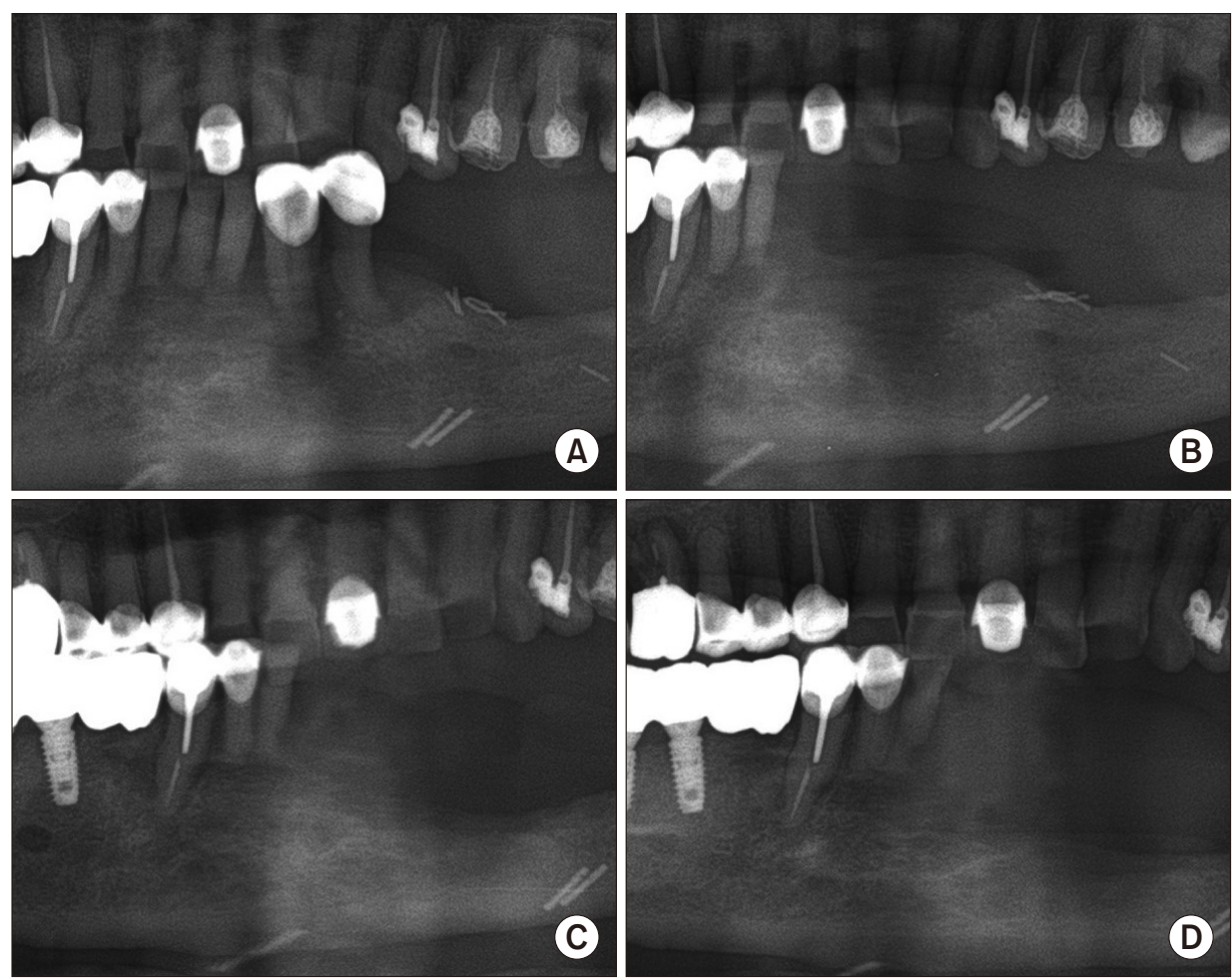

Fig. 3. Serial radiographic changes after using pentoxifylline and tocopherol in the Case No. 7 patient who began medications after surgical wound healing (56 days). A. Before surgical treatment. B. After surgery including extraction and saucerization and before beginning medications. C. Three months after drug administration. D. Six months after drug administration with the figure showing an extended osteoIytic lesion.

Mi Hyun Seo et al: The effects of pentoxifylline and

D tocopherol in jaw osteomyelitis. J Korean Assoc Oral Maxillofac Surg 2020

In the result of the ratio of densities of ROI compared to the contralateral site, there was no statistical significance between disease group and duration of drug administration $(P>0.05)$. Due to radiographic characteristics after bone healing, the radiographic densities were similar to that of the surrounding bone, not contralateral side. In BRONJ, sclerotic changes were more often observed compared to that in ORN and osteomyelitis. After the bone healing period, radiographic densities further increased compared to the reference contralateral site.

Typically, the first choice for diagnostic imaging of osteonecrosis or osteomyelitis is the dental periapical view and panoramic radiography. Panoramic radiography is relatively easy to perform, is inexpensive and convenient, and exposes the patient to the lowest radiation dose ${ }^{14}$. Initial changes of ORN manifest in subtle areas of deformed trabecular struc- 

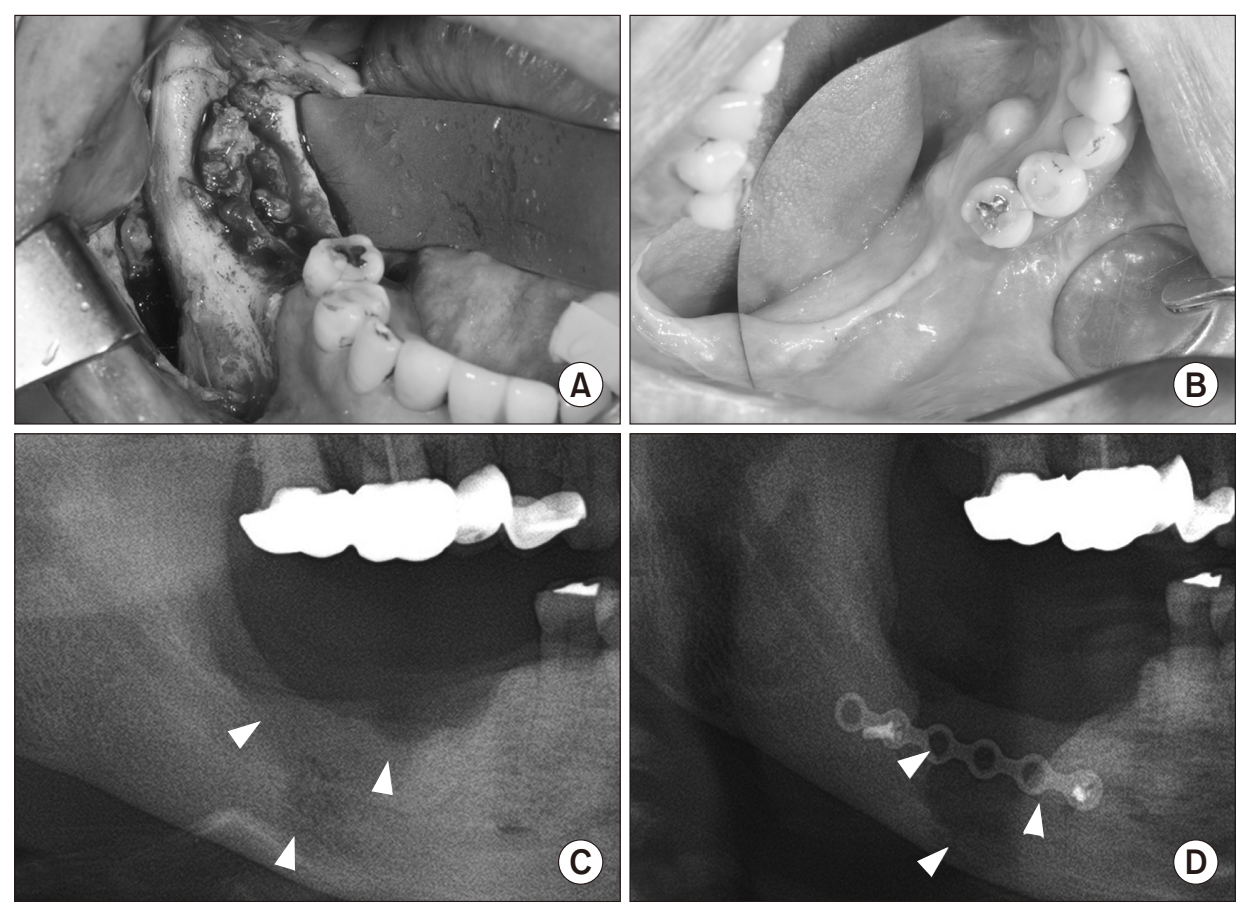

Fig. 4. Serial radiographic changes with clinical photos after using pentoxifylline (PTX) and tocopherol in the Case No. 10 patient who began PTX and tocopherol after surgical wound healing for treatment of bisphosphonate-related osteonecrosis of the jaw (BRONJ) for 8 months. A. Clinical picture showing
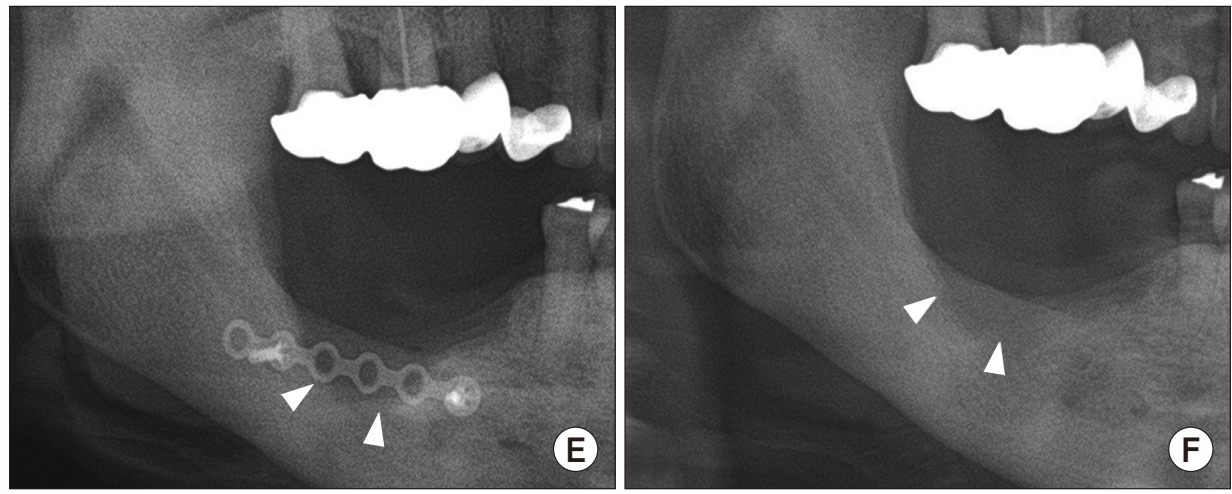
the pathologic lesion before surgery. B. One year after surgery and drug administration. C. Preoperative panorama showing the lesion extending to the mandibular border. D. One week after surgery, at the beginning treatment of PTX and tocopherol. E. Six months after drug administration. F. One year after drug administration. (Arrowheads: radiolucent lesion of $\mathrm{BRONJ}$ in the panoramic views)

Mi Hyun Seo et al: The effects of pentoxifylline and tocopherol in jaw osteomyelitis. J Korean Assoc Oral Maxillofac Surg 2020

Table 2. Comparison of changes in radiographic densities according to duration of pentoxifylline (PTX) and tocopherol use

\begin{tabular}{cccc}
\hline & $\begin{array}{c}\text { More than 90 } \\
\text { days }(\mathrm{n}=17)\end{array}$ & $\begin{array}{c}\text { Less than 90 days } \\
(\mathrm{n}=8)\end{array}$ & $P$-value \\
\hline$\Delta 1$ (T1-T0) & $2.86 \pm 8.62$ & $2.52 \pm 7.06$ & 0.97 \\
$\Delta 2$ (T2-T1) & $4.96 \pm 6.77$ & $-6.81 \pm 5.02$ & 0.013 \\
$\Delta 3$ (T2-T0) & $7.24 \pm 10.03$ & $-5.20 \pm 6.78$ & 0.006 \\
\hline
\end{tabular}

(T0: before taking PTX and tocopherol, T1: 3 months after taking PTX and tocopherol, T2: 6 months after taking PTX and tocopherol, $\Delta 1$ (T1-T0): difference of radiographic densities between $\mathrm{T} 1$ and $\mathrm{T} 0$, $\Delta 2$ (T2-T1): difference of radiographic densities between $\mathrm{T} 2$ and $\mathrm{T} 1$, $\Delta 3$ (T2-T0): difference of radiographic densities between T2 and T0) Statistical significance test was performed with the Mann-Whitney U test.

Values are presented as mean \pm standard deviation.

Mi Hyun Seo et al: The effects of pentoxifylline and tocopherol in jaw osteomyelitis. $J$ Korean Assoc Oral Maxillofac Surg 2020

tures with rarefaction. As the disease progresses, osteolytic bone destruction becomes radiographically evident and produces a patchy radiolucent appearance with radiodense is-
Table 3. Comparison of the time and disease differences of the radiation densities between the region of interest and the contralateral side

\begin{tabular}{lllr}
\hline & \multicolumn{1}{c}{ ORN } & BRONJ & \multicolumn{1}{c}{ OM } \\
\hline Per $\Delta 0$ & $22.71 \pm 15.67$ & $14.14 \pm 9.25$ & $12.20 \pm 9.58$ \\
$\operatorname{Per} \Delta 1$ & $13.44 \pm 9.05$ & $11.34 \pm 7.13$ & $10.72 \pm 8.75$ \\
$\operatorname{Per} \Delta 2$ & $12.20 \pm 9.58$ & $10.01 \pm 9.30$ & $9.30 \pm 8.83$ \\
\hline
\end{tabular}

(Per $\Delta 0$ : ratio of radiographic densities before taking pentoxifylline [PTX] and tocopherol, Per $\Delta 1$ : ratio of radiographic densities 3 months after taking PTX and tocopherol, Per $\Delta 2$ : ratio of radiographic densities 6 months after PTX and tocopherol, ORN: osteoradionecrosis, BRONJ: bisphosphonate-related osteonecrosis of the jaws, OM: chronic osteomyelitis)

Statistical significance test was performed with repeated measures ANOVA.

Values are presented as mean \pm standard deviation.

Mi Hyun Seo et al: The effects of pentoxifylline and tocopherol in jaw osteomyelitis. $J$ Korean Assoc Oral Maxillofac Surg 2020

lands of necrotic bone or sequestrum. In BRONJ, radiographic changes include osteolytic and sclerotic lesions. While the 
Table 4. Changes in ESR over time

\begin{tabular}{lll}
\hline & \multicolumn{1}{c}{ Pre } & \multicolumn{1}{c}{ Post } \\
\hline ORN & $28.67 \pm 25.58$ & $19.00 \pm 15.40$ \\
BRONJ & $32.25 \pm 14.14$ & $25.00 \pm 20.14$ \\
OM & $16.40 \pm 10.57$ & $11.00 \pm 7.62$ \\
Total & $26.63 \pm 16.22^{*}$ & $19.50 \pm 16.63^{*}$ \\
\hline
\end{tabular}

(ESR: erythrocyte sedimentation rate, ORN: osteoradionecrosis, BRONJ: bisphosphonate-related osteonecrosis of the jaw, OM: chronic osteomyelitis, Pre: initial ESR, Post: ESR at 3 to 6 months after PTX and tocopherol administration)

$* P<0.05$; Statistical significance test was performed with repeated measures ANOVA.

Values are presented as mean \pm standard deviation.

Mi Hyun Seo et al: The effects of pentoxifylline and tocopherol in jaw osteomyelitis. $J$ Korean Assoc Oral Maxillofac Surg 2020

radiographic appearances of ORN and BRONJ may overlap, the prominent activation of osteoclasts and the attenuation of osteoblastic function during ORN result in the rarefying appearance with loss of cortical outlines and trabecular density. In contrast, osteoclastic inhibition in BRONJ resulted in an overall increase in density of the trabeculae and cortex ${ }^{15}$. In our results, there were no statistical differences between the disease groups. However, in the BRONJ group, radiographic densities increased after wound healing compared to the contralateral site. ORN exhibits predominantly osteolytic lesions on radiographs, while sclerotic lesions are frequently observed with BRONJ. Changes in the degree of densities on $\mathrm{x}$-rays are greater for ORN lesions even though there is no statistical significance.

Unfortunately, there are no long-term follow-up patients after discontinuation of PTX and tocopherol for therapeutic benefit. Of the 8 patients who took drugs for short-term period, five patients were treated with chronic osteomyelitis and BRONJ. Even after the drug was withdrawn, they showed stable bone healing. One of the other three patients in the ORN group showed same radiolucent lesion in the panorama one year after drug cessation. He started PTX and tocopherol treatment again. The rebound effect after drug withdrawal may be related to the type of disease and the extent of the lesion.

Laboratory data including $\mathrm{WBC}, \mathrm{Hb}$, Hct, ANC, and ESR values were collected at the initial hospital visit. Statistically significant differences were found in $\mathrm{Hb}$ between the ORN and BRONJ groups $(P=0.016)$. The mean value of $\mathrm{Hb}$ in BRONJ patients was lowest of the groups since most of the patients were older women suffering from osteoporosis. Among the inflammatory laboratory markers, only ESR exhibited statistically significant change with time. However, there were no differences between ORN, BRONJ, and chronic osteomyelitis. Therefore, recovery of ESR to normal range may be considered as an index to evaluate successful treatment of osteomyelitis.

Established radiation induced bony damage is known to be irreversible. However, Delanian and Lefaix ${ }^{16}$ suggested that radiation induced fibrosis could be reversed by antioxidant therapy with PTX, tocopherol, and clodronate. Delanian et al. ${ }^{6}$ published a prospective clinical trial of 18 patients diagnosed with mandibular refractory ORN who had failed to improve after at least two months of conventional treatment. Sixteen of the 18 patients with ORN had complete mucosal healing with a median time to recovery of six months. Delanian et al. ${ }^{7}$ developed an impressive regimen (PENTOCLOPENtoxifylline-TOcopherol-CLOdronate) in a refractory group of 54 patients. The amount of exposed bone was reduced by $42 \%$ ( 2 months), 62\% (4 months), 77\% (6 months), $92 \%$ (12 months), and 96\% (18 months). McLeod et al. ${ }^{17}$ reported a retrospective study of 12 patients who received PTX and tocopherol with improvement in five patients, no change in five patients, and worsening in two patients. However, three patients whose final score improved after operations despite progression of ORN while on PTX and tocopherol underwent radical resection and reconstruction. In a retrospective study of 71 patients treated for ORN of the mandible after medical management composed of PTX, tocopherol, and doxycycline, the patients who required resection and free flap reconstruction declined from 51\% (20/39) to $25 \%$ $(8 / 32)^{18}$.

In a study of six patients in the field of BRONJ treatment, all the patients with BRONJ after treatment with PTX and tocopherol exhibited improved symptoms by an average reduction of $74 \%$ in bone exposed areas ${ }^{19}$. ONJ from osteoporotic patients who underwent yearly zoledronic acid administration and were concomitantly treated with corticosteroids was effectively managed with PTX and tocopherol ${ }^{20}$. In a recent case series, all seven patients suffering from ONJ demonstrated symptom relief, while four patients exhibited bony regeneration of radiolucent defects ${ }^{21}$.

In this clinical study, two patients (Cases No. 2 and No. 4) were diagnosed with ORN and received only medical treatment with PTX and tocopherol without any surgical intervention. Those patients did not experience any adverse effects due to the medication. Clinical photos and serial panoramic views show mucosal coverage and gradient bone occupying the defect.(Fig. 2)

Generally, established ORN, BRONJ, and chronic osteomyelitis do not regress spontaneously. Pathologic lesions 
can be stabilized or gradually worsen and can often be enlarged to such an extent that management becomes difficult. PTX has been reported to reduce radiation induced fibrosis. The mechanism of inhibiting fibrosis or reversing fibrosis has yet to be elucidated ${ }^{22}$. PTX has been shown to enhance microvascular blood flow and reduce platelet aggregation to maintain perfusion in radiated tissue. PTX also downregulates the production of proinflammatory cytokines, particularly TNF- $\alpha$, in response to noxious stimuli and inhibits granulocyte-mediated cytotoxicity after TNF- $\alpha$ exposure and provides protection against radiation-induced, cytokinemediated cellular damage ${ }^{23}$. PTX and tocopherol can be used as an auxiliary to surgery for the treatment of ORN, BRONJ, and chronic osteomyelitis. Unfortunately, there is no guideline for how long PTX and tocopherol should be taken. In a study by Delanian et al. ${ }^{24}$, two-thirds of patients responded to a maximal response after an average of two years. They recommended that long term treatment of more than three years in the presence of severe radiation induced fibrosis because of the risk of rebound effects. In this study, most patients were treated with primary surgery such as curettage, sequestrectomy, and saucerization. Drugs have been shown to increase bone filling and stabilize pathological conditions of the mandible when used over an extended period of three months or more. Further long-term studies involving large numbers of patients with additional inflammation markers, such as C-reactive protein and procalcitonin, are needed.

\section{Conclusion}

This clinical study of 25 patients with ORN, BRONJ, or chronic osteomyelitis demonstrated the clinical effects of PTX and tocopherol. The long-term use of the combination of PTX and tocopherol for more than 3 months exhibited positive effects on bone healing in BRONJ, chronic osteomyelitis, or ORN. Although surgery preceded drug administration, PTX and tocopherol may be used as an adjunctive treatment. Additional research is needed to support the potential role of pharmacologic therapy in the progression of these diseases.

\section{ORCID}

Mi Hyun Seo, https://orcid.org/0000-0001-8220-6480

Mi Young Eo, https://orcid.org/0000-0001-7055-9924

Hoon Myoung, https://orcid.org/0000-0002-9984-8479

Soung Min Kim, https://orcid.org/0000-0002-6916-0489

Jong Ho Lee, https://orcid.org/0000-0002-8843-545X

\section{Authors' Contributions}

M.H.S. participated in data collection and wrote the manuscript, M.Y.E. participated in the study design and performed the statistical analysis, H.M. participated in the study design, S.M.K. revised and wrote the manuscript, and J.H.L. participated in the study design. All authors read and approved the final manuscript.

\section{Acknowledgements}

This study was supported by a grant of the Korean Health Technology R\&D Project, Ministry of Health \& Welfare, Republic of Korea (HI15C0689), and by Basic Science Research Program through the NRF funded by the Ministry of Education (2017R1D1A1B04029339).

\section{Ethics Approval and Consent to Participate}

All procedures followed were in accordance with the ethical standards of the responsible committee (institutional and national) and with the Helsinki Declaration of 1964 and later versions. Informed consent was not required in this study. The collection and processing of this retrospective clinical data were approved by the Institutional Review Board (IRB No. S-D20160041) of Seoul National University.

\section{Conflict of Interest}

No potential conflict of interest relevant to this article was reported.

\section{References}

1. Martos-Fernández M, Saez-Barba M, López-López J, EstrugoDevesa A, Balibrea-Del-Castillo JM, Bescós-Atín C. Pentoxifylline, tocopherol, and clodronate for the treatment of mandibular osteoradionecrosis: a systematic review. Oral Surg Oral Med Oral Pathol Oral Radiol 2018;125:431-9.

2. Ward A, Clissold SP. Pentoxifylline. A review of its pharmacodynamic and pharmacokinetic properties, and its therapeutic efficacy. Drugs 1987;34:50-97.

3. Magnusson M, Gunnarsson M, Berntorp E, Björkman S, Höglund P. Effects of pentoxifylline and its metabolites on platelet aggregation in whole blood from healthy humans. Eur J Pharmacol 2008;581:290-5.

4. Neuner P, Klosner G, Schauer E, Pourmojib M, Macheiner W, Grünwald $\mathrm{C}$, et al. Pentoxifylline in vivo down-regulates the release of IL-1 beta, IL-6, IL-8 and tumour necrosis factor-alpha by human peripheral blood mononuclear cells. Immunology 1994;83:262-7.

5. Lyons AJ, Brennan PA. Pentoxifylline: a review of its use in osteo- 
radionecrosis. Br J Oral Maxillofac Surg 2017;55:230-4.

6. Delanian S, Depondt J, Lefaix JL. Major healing of refractory mandible osteoradionecrosis after treatment combining pentoxifylline and tocopherol: a phase II trial. Head Neck 2005;27:114-23.

7. Delanian S, Chatel C, Porcher R, Depondt J, Lefaix JL. Complete restoration of refractory mandibular osteoradionecrosis by prolonged treatment with a pentoxifylline-tocopherol-clodronate combination (PENTOCLO): a phase II trial. Int J Radiat Oncol Biol Phys 2011;80:832-9.

8. Qaisi M, Montague L. Bone margin analysis for osteonecrosis and osteomyelitis of the jaws. Oral Maxillofac Surg Clin North Am 2017;29:301-13.

9. Notani K, Yamazaki Y, Kitada H, Sakakibara N, Fukuda H, Omori $\mathrm{K}$, et al. Management of mandibular osteoradionecrosis corresponding to the severity of osteoradionecrosis and the method of radiotherapy. Head Neck 2003;25:181-6.

10. Ruggiero SL, Dodson TB, Fantasia J, Goodday R, Aghaloo T, Mehrotra B, et al. American Association of Oral and Maxillofacial Surgeons position paper on medication-related osteonecrosis of the jaw--2014 update. J Oral Maxillofac Surg 2014;72:1938-56.

11. Ruggiero SL. Diagnosis and staging of medication-related osteonecrosis of the jaw. Oral Maxillofac Surg Clin North Am 2015;27:479-87.

12. Futran ND, Trotti A, Gwede C. Pentoxifylline in the treatment of radiation-related soft tissue injury: preliminary observations. Laryngoscope 1997;107:391-5.

13. Zhao Y, Liu B, Wang SP, Wang YN. Computed densitometry of panoramic radiographs in evaluation of bone healing after enucleation of mandibular odontogenic keratocysts. Chin J Dent Res 2010;13:123-6.

14. White SC, Mallya SM. Update on the biological effects of ionizing radiation, relative dose factors and radiation hygiene. Aust Dent $\mathrm{J}$ 2012;57 Suppl 1:2-8.

15. Mallya SM, Tetradis S. Imaging of radiation- and medicationrelated osteonecrosis. Radiol Clin North Am 2018;56:77-89.

16. Delanian S, Lefaix JL. The radiation-induced fibroatrophic process: therapeutic perspective via the antioxidant pathway. Radiother Oncol 2004;73:119-31.

17. McLeod NM, Pratt CA, Mellor TK, Brennan PA. Pentoxifylline and tocopherol in the management of patients with osteoradionecrosis, the Portsmouth experience. Br J Oral Maxillofac Surg
2012;50:41-4.

18. D'Souza J, Lowe D, Rogers SN. Changing trends and the role of medical management on the outcome of patients treated for osteoradionecrosis of the mandible: experience from a regional head and neck unit. Br J Oral Maxillofac Surg 2014;52:356-62.

19. Epstein MS, Wicknick FW, Epstein JB, Berenson JR, Gorsky M. Management of bisphosphonate-associated osteonecrosis: pentoxifylline and tocopherol in addition to antimicrobial therapy. An initial case series. Oral Surg Oral Med Oral Pathol Oral Radiol Endod 2010;110:593-6.

20. Magremanne M, Reychler H. Pentoxifylline and tocopherol in the treatment of yearly zoledronic acid-related osteonecrosis of the jaw in a corticosteroid-induced osteoporosis. J Oral Maxillofac Surg 2014;72:334-7.

21. Owosho AA, Estilo CL, Huryn JM, Yom SK. Pentoxifylline and tocopherol in the management of cancer patients with medicationrelated osteonecrosis of the jaw: an observational retrospective study of initial case series. Oral Surg Oral Med Oral Pathol Oral Radiol 2016;122:455-9.

22. Boerma M, Roberto KA, Hauer-Jensen M. Prevention and treatment of functional and structural radiation injury in the rat heart by pentoxifylline and alpha-tocopherol. Int J Radiat Oncol Biol Phys 2008;72:170-7.

23. Rübe CE, Wilfert F, Uthe D, Schmid KW, Knoop R, Willich N, et al. Modulation of radiation-induced tumour necrosis factor alpha (TNF-alpha) expression in the lung tissue by pentoxifylline. Radiother Oncol 2002;64:177-87.

24. Delanian S, Porcher R, Rudant J, Lefaix JL. Kinetics of response to long-term treatment combining pentoxifylline and tocopherol in patients with superficial radiation-induced fibrosis. J Clin Oncol 2005;23:8570-9.

How to cite this article: Seo MH, Eo MY, Myoung H, Kim SM, Lee JH. The effects of pentoxifylline and tocopherol in jaw osteomyelitis. J Korean Assoc Oral Maxillofac Surg 2020;46:19-27. https://doi.org/10.5125/jkaoms.2020.46.1.19 